\title{
Nouvelles approches des relations producteurs et consommateurs
}

Oléagineux, Corps Gras, Lipides. Volume 8, Numéro 6, 584-6, Novembre - Décembre 2001, Dossier : L'avenir des cultures pérennes

Auteur(s) : Philippe CHALMIN, EULER-sfac, 1, rue Euler, 75008 Paris.

\section{ARTICLE}

Je voudrais profiter du pouvoir de la présidence pour tenter de recadrer un certain nombre de choses et de problématiques qui ont été évoquées ici et là au cours de ces journées.

Pour bien comprendre la situation dans laquelle nous nous trouvons aujourd'hui, un peu d'histoire est nécessaire. Remontons à 1976, à l'époque de la résolution 93/4 de la $4^{\mathrm{e}}$ Cnuced de Nairobi. Tous ceux qui l'ont vécue s'en souviennent encore. Ce fut un moment tout à fait fondamental auquel nous avons tous cru et qui vit l'adoption du programme intégré des produits de base, programme qui devait servir de cadre aux relations internationales des dix années suivantes. Ce fut aussi l'époque du " choc des matières premières", de l'emploi de "l'arme alimentaire ". Un certain nombre de pays producteurs s'initièrent ainsi aux techniques de marchés, afin de peser sur les cours. Souvenez-vous du groupe de Bogota. Simultanément au Nord et au Sud, des politiques agricoles furent mises en place ou renforcées. Bref, cette époque, celle des années 70, fut résolument celle de l'optimisme. Nous vivions, ne l'oublions pas, la deuxième décennie du développement, après les fameuses «Trente Glorieuses ».

Tout cela ne dura guère, il faut bien le reconnaître. Et une décennie plus tard, vers le milieu des années 80 , quel constat devions-nous faire ? Les accords internationaux débouchaient les uns après les autres sur des échecs plus ou moins retentissants. L'accord sur l'étain, celui sur le café, le projet des cartellisations étaient tour à tour abandonnés. Bref, nous assistions à l'effondrement de ce programme intégré des produits de base sur lequel nous avions fondé tant d'espoirs et qui, en guise de développement, avait surtout favorisé l'essor de l'hôtellerie genevoise. Se révélait également l'échec relatif, ou du moins l'escalade importante, des coûts des politiques agricoles des pays du Nord (politique agricole européenne, Farm Act nord-américain) et des dispositifs nationaux mis en place dans un certain nombre de pays du Sud. Un constat qui signifiait la fin du développement traditionnel, du développement auto-centré, sur lequel nous avions fondé, là aussi, quelques espoirs et qui débouchait sur les grandes crises d'endettement d'un certain nombre de pays du tiers monde.

Puis arrivèrent les années 90 , et avec elles apparurent un nouveau paradigme et un nouveau vocabulaire. On se mit à parler de mondialisation, de nouvelle économie, de libéralisme. Au fond, si je résume, l'euphorie qui gagna les esprits, ces années-là, exprimait pour l'essentiel les perspectives heureuses qu'était censé apporter le mariage de la puce informatique et du marché. Survinrent effectivement un développement économique mondial extrêmement important et une période de croissance de plus de dix ans comme les États-Unis d'Amérique n'en avaient jamais connus et qui vient de se terminer. Apparut dans le même temps un modèle de développement inattendu, le modèle asiatique, largement extraverti et qui permit le décollage de tout un continent. Bref, la foi dans les vertus du marché et de sa main invisible ne cessait de se répandre de par le monde. 
Au Nord comme au Sud, des programmes de dérégulations et de privatisations furent lancés qui touchèrent même le domaine agricole, traditionnellement protégé des vagues libérales. Il y eut ainsi la remise en cause, très modeste j'en conviens, des politiques agricoles des pays du Nord, et qui n'eut que de maigres résultats malgré les nombreux différends portés devant le Gatt puis devant l'OMC. Et furent lancés parallèlement les grands programmes d'ajustements structurels, de libéralisation des politiques agricoles des pays du Sud. Et, il faut bien le reconnaître, ces derniers devinrent le terrain de chasse privilégié des libéraux, souvent les plus doctrinaires qui, frustrés de ne pas voir leurs recettes appliquées au Nord, exercèrent tout leur zèle pour tirer à vue sur les caisses, les boards, et autres dispositifs de cet acabit. Nous avons pu constater, encore tout à l'heure, combien la Banque mondiale se félicite d'avoir " tué » les boards de l'Ouganda, tout en gardant un silence résigné sur l'existence des boards céréaliers qui continuent de prospérer au Canada et en Australie.

Cette période fut également celle de la " commoditisation " de très nombreux produits et services. Cette notion de commodité - mauvaise traduction française du terme anglo-saxon de commodities s'applique désormais non seulement aux produits financiers mais aussi à de très nombreux produits industriels : les puces informatiques en sont le plus bel exemple. Nous sommes entrés dans une phase de commoditisation d'un certain nombre de services comme l'électricité et l'énergie au sens le plus large, mais aussi de services, comme la minute d'impulsion téléphonique.

Reconnaissons que cette époque d'euphorie a connu quelques résultats, quoiqu'on en dise. Des pays ont effectivement décollé et ont symptomatiquement réussi à se défaire de la malédiction des matières premières. On cite la Malaisie, on pourrait peut-être ajouter la Thaïlande. La liste n'en est point très longue. Car il est vrai que ces roaring nineties, ces brillantes années 90 , ont également été pour nombre de pays celles de l'instabilité des marchés des produits de base, celles de l'absence de développement, voire celles de la poursuite du sous-développement.

Cette foi aveugle, qui a été un peu la nôtre durant ces années-là, a pris fin - et avec elle le $x^{\mathrm{e}}$ siècle avec les attentats du World Trade Center, le 11 septembre 2001 à 15 h GMT.

Cette périodisation est très commode : elle permet à tous les économistes comme moi qui étaient en train de se tromper en termes de croissance, de gommer leurs positions et de dire qu'ils n'y sont pour rien, qu'il y a eu choc externe. Or, nous savions que nous étions déjà entrés dans une phase de récession économique. II y avait déjà eu un krach boursier et nous savions qu'il y en aurait probablement d'autres. Certes, ce n'était pas dramatique, mais c'était quand même une première atteinte à cette foi mise dans les vertus du marché et d'une économie mondiale libéralisée.

Il est frappant de constater combien l'une des conséquences du 11 septembre a été un peu partout la réhabilitation du rôle de l'État et la prise de conscience de la nécessité de donner au politique un rôle plus important. On peut même supposer - sans trop pécher par optimisme - que d'une certaine façon fut ressenti combien c'est tout le problème du développement qui se profile derrière ces attentats, bref que nous avons besoin d'un nouvel élan de solidarité au niveau mondial comme celui que les mouvements dits d'anti-mondialisation étaient les seuls à promouvoir jusqu'à aujourd'hui.

Et il est vrai qu'un nouveau dialogue entre producteurs et consommateurs n'est jamais apparu autant nécessaire. Les marchés des produits qui nous intéressent, et d'autres, sont au plus bas. Le café connaît son plus bas niveau historique, et le cacao n'est guère plus brillant. Le caoutchouc est presque dans le même cas. Et si pour ce dernier, on peut effectivement incriminer la baisse de la demande due 
à la récession industrielle, pour l'huile de palme, c'est probablement beaucoup plus la politique américaine de subvention du soja qui est en cause. Les filières sont ainsi en plein désarroi. L'exemple de la Côte d'Ivoire avec le cacao est, de ce point de vue, édifiant. On pourrait le retrouver dans nombre d'autres pays du tiers monde.

Enfin, pour souligner combien il est temps d'agir et combien le libéralisme pur et dur est peut-être aujourd'hui un peu moins sûr de son fait, rappelons que la récente attribution du prix Nobel d'économie à Joseph Stiglitz (un dissident de la Banque mondiale) n'est pas totalement neutre. II vient de rappeler dans un de ses premiers articles publiés après son prix, l'importance du rôle de l'État et de la fonction régalienne, $\mathrm{y}$ compris dans un certain nombre de services.

Sur ce constat j'aimerais maintenant lancer la discussion et tenter d'identifier de nouvelles approches. C'est là évidemment un exercice beaucoup plus difficile, car sur le fond que peut-il y avoir de nouveau?

Je propose que nous organisions notre discussion en deux parties. Nous aborderons dans un premier temps les mesures qu'il est possible d'envisager sur le plan international. Puis, satisfaits ou non des propositions brillantes que nous aurons trouvées à l'international, nous tenterons, dans une deuxième partie, de reformuler le problème sur le plan national...

Je vois fondamentalement quatre familles de solutions, quatre familles d'approche sur le plan international.

La première consiste à revendiquer le marché, rien que le marché et tout le marché. Je suis un horrible libéral, c'est entendu, un libéral pur et dur, mais cela présente quand même quelques avantages à long terme : le marché, tout aveugle qu'il soit, est relativement efficient. Cela étant, pour bien fonctionner, il n'en exige pas moins des conditions de transparence, de contrôle et de bonne gouvernance rarement réunies au niveau international. Les anti-mondialisation ont raison de s'en prendre aux paradis fiscaux, à la corruption internationale, aux entraves à la concurrence, aux ententes des forts contre les faibles. Les marchés internationaux tels que nous les connaissons sont loin d'être parfaits et peut-être quelque autorité d'arbitrage leur donnerait une plus grande efficacité. Mais ne nous voilons pas la face : le marché des produits agricoles crée de l'instabilité, celle-ci est inévitable. Au xviiie siècle, l'abbé Galliani a publié un des premiers ouvrages d'économie politique, un dialogue sur les commerces des blés, et on n'a depuis lors, me semble-t-il, rien inventé de nouveau, sinon des modèles pour expliquer le passé, et se tromper sur l'avenir. L'instabilité est là. Et quand on est en situation d'instabilité, quand on est producteur, on se trouve, appelons un chat un chat, dans une situation de spéculateur. Tout producteur de matière première de plantes pérennes, est quelqu'un qui est en permanence en situation de spéculateur et qui doit donc anticiper : speculare, en latin signifie regarder dans le lointain. II ne faut pas l'oublier. C'est la grandeur, ce sont aussi les limites, du marché : tout le monde peut couvrir ces risques, sauf les deux bouts de la filière.

Dans ces conditions, il est possible - deuxième famille de solutions - de recourir aux techniques des marchés dérivés pour tenter, non pas de supprimer cette spéculation, mais de l'optimiser. Autrement dit, proposons aux producteurs de couvrir eux-mêmes une partie de leurs risques ! Je veux bien ! Je remarque simplement qu'aux États-Unis, face à une solution de ce genre subventionnant les options, les agriculteurs ont jugé préférable, car plus sûr, d'être directement subventionnés par l'État. De 
toutes manières, l'amélioration envisagée ne serait que marginale et contribuerait au mieux à une meilleure transparence, soit encore peu de chose.

On peut aussi se tourner - troisième famille de solutions - vers les techniques traditionnelles de gestion de l'offre. Peut-on réhabiliter les cartels ? Je ne sais ce qu'en pensent les producteurs de café et de cacao. Le système de quota a plus ou moins bien fonctionné par le passé : même l'accord du café était devenu à la fin des années 80 une vraie passoire. Le système ne fonctionnait que parce qu'il y avait le hors quota. Peut-on imposer - ce qui a été proposé tout à l'heure par un intervenant dans la salle - un prix minimum garanti mondial, en supposant que le consommateur et le producteur soient capables de s'entendre sur un seuil minimal ? Ces techniques ont été largement utilisées depuis trente ans, avec les résultats que l'on sait.

Demeure un quatrième ensemble de possibilités. Il est difficile de le qualifier, mais j'ai entendu beaucoup de mots ici et là exprimant ce qu'il recouvre. Le mot coopération, par exemple : peut-on aller vers une plus grande coopération au sein des filières ? Peut-être. Mais sur le long terme, la contractualisation peut se révéler dangereuse, car trop déséquilibrée. J'ai entendu l'expression de " commerce équitable "... Je m'interroge. Pourquoi celui-ci serait-il plus équitable que l'autre ? Les prix du café ou du cacao aujourd'hui ne sont pas inéquitables : ils sont la simple résultante du jeu de l'offre et de la demande. Mais il est clair qu'en continuant sur ce registre, on s'éloigne progressivement du champ de l'économie. L'« équité ", le " juste " prix : très franchement je ne vois pas, bien qu'économiste, ce que ces termes pourraient recouvrir. On est déjà sur le terrain de la morale. De toute façon, rien n'interdit d'innover. J'ai entendu hier proclamer : «Planteurs et industriels du monde, unissez-vous "... pourquoi pas ? Les prix du café et du cacao peuvent très bien relever de la solidarité internationale. Ces deux produits ne sont pas essentiels pour nos économies du Nord... et bien engageons-nous à payer un prix minimum ! Est-ce une utopie ? D'un point de vue économique, certainement, car encore une fois sur ce terrain ces notions n'ont rigoureusement aucun sens. Mais peut-être doit-on quitter le champ de l'économie. II est vrai que d'une certaine façon, aujourd'hui, le prix du café est un prix immoral. Est-ce que la communauté des nations est prête à un geste de solidarité ? C'est une question que je pose et c'est ce que l'intervenant précédent évoquait quand il demandait l'adoption d'un prix minimum mondial. Pourquoi pas ? Mais nous sommes désormais dans le champ du politique. Peut-être que le 11 septembre nous oblige à réfléchir dans ce sens. Néanmoins, je perçois très bien la boîte de Pandore que l'on s'apprête ainsi à ouvrir et le type de problèmes qui pourraient en sortir. Jean-Marc Boussard tout à l'heure a d'ailleurs résumé à sa façon cette proposition quand il a demandé que l'on se passe du marché !

Voilà au plan international les sujets de réflexion que je verse aux débats. Je n'ai peut-être pas suffisamment d'imagination. Je suis sûr que vous en aurez pour moi.

Sur le plan national, je vois trois orientations possibles.

Je crois tout d'abord qu'il est important de rappeler que les pays du Sud ont le droit d'avoir des politiques agricoles. Que les bien-pensants de la Banque mondiale ou d'ailleurs qui le prendraient mal soient ici renvoyés à l'étude de la politique agricole européenne ou à celle des dérives du Farm Act américain ces quatre dernières années. Comment peut-on encore s'opposer - en particulier pour le domaine des produits vivriers - aux mesures de protection des marchés intérieurs ou, pour reprendre le titre d'un livre de mon ami Bertrand Hervieu, président de l'Inra, aux " droits des peuples à se nourrir eux-mêmes " ? La politique agricole est un des instruments de votre souveraineté nationale... 
c'est celui que nous avons utilisé depuis cinquante ans au Nord et, au fond, avec pas mal de succès. Demeure néanmoins la question des produits des filières d'exportation : nous touchons ici au cœur du problème, particulièrement en Côte d'Ivoire. Quel rôle assigner à l'État ? Faut-il, à coups d'ajustements structurels, laisser disparaître toute idée d'intervention publique ? Je n'en suis pas certain. Je crois au contraire en la nécessité d'une politique de gestion des filières d'exportation. Seul un État fort peut être le garant nécessaire de la transparence et de l'équité du marché. Et ce n'est pas contrevenir aux professions de foi libérales que de rappeler que seul un État doté des moyens financiers dont il aura su éventuellement se pourvoir, pourra garantir un niveau de prix minimum et par là même un minimum de stabilité à ses planteurs - stabilité dont bénéficient les producteurs européens et nord-américains depuis soixante ans maintenant ! Lorsque j'entends dire que le paysan ivoirien pourra regarder son journal et puis acheter les options sur la base des prix à New York, alors que je suis incapable de proposer ça à un producteur de la Beauce français, qu'on ne me fasse pas rigoler : ça ne marchera pas. Je crois que l'État a un rôle important à jouer. En fonction des traditions historiques, de l'équilibre de la production, des structures existantes, ce rôle pourra s'exprimer de manière différente. Et puis, on peut avancer à petits pas vers une économie de la régulation, afin de marier l'existence du marché avec un filet de sécurité assuré par l'État. Nous avons là une véritable réflexion de fond à mener. Comment optimiser la gestion d'une filière d'exportation ? Probablement en taxant moins : la Banque mondiale a raison ; certainement aussi en protégeant, surtout lorsqu'on connaît l'environnement - je pense là surtout au café et cacao - des petits producteurs. Je rappelle que la Malaisie s'est développée avec le caoutchouc et l'huile de palme, dans le cadre d'une organisation largement parapublique, dans laquelle les agriculteurs étaient tous totalement engagés. Donc, nous avons là une deuxième réflexion tout à fait importante à mener.

La troisième piste au plan national, le professeur Dosso en a parlé tout à l'heure, c'est celle de la diversification. Chris Gilbert a bien souligné qu'il ne s'agit pas de diversifier les productions agricoles mais bien de faire autre chose. La clé du succès malais c'est d'avoir développé une économie industrielle et je crois que là est la véritable question. Que faire pour changer l'axe général de I'orientation du développement, surtout en Afrique?

Pour conclure, et avant de donner la parole aux différents intervenants de cette table ronde, je terminerai avec deux questions provocantes. Comme elles sont provocantes, elles n'appellent peutêtre pas de réponse ; je les livre néanmoins à votre réflexion. La première est celle-ci : qu'est-ce qu'un juste prix ? La réponse est chez Saint Thomas d'Aquin (pour ceux que cela intéresse). La deuxième enveloppe tous les travaux de ce colloque et pourrait se formuler ainsi : la malédiction des matières premières est-elle irréversible ? Relisez à ce sujet les travaux de Kaldor et Thinberghen : malheureusement la réponse n'est pas simple...

Voilà les quelques mots que je souhaitais prononcer en guise d'introduction. Non sans avoir totalement abusé, je m'en rends compte, de mon privilège présidentiel.

(Recueilli par Jean-Claude Icart)

* Propos introductifs à la table ronde « Nouvelles approches entre producteurs et consommateurs", Yamoussoukro, 6 novembre 2001. 